\title{
A NOTE ON DERIVATIONS OF LIE ALGEBRAS
}

\author{
M. SHAHRYARI
}

(Received 12 February 2011)

\begin{abstract}
In this note, we will prove that a finite-dimensional Lie algebra $L$ over a field of characteristic zero, admitting an abelian algebra of derivations $D \leq \operatorname{Der}(L)$, with the property

$$
L^{n} \subseteq \sum_{d \in D} d(L)
$$

for some $n>1$, is necessarily solvable. As a result, we show that if $L$ has a derivation $d: L \rightarrow L$ such that $L^{n} \subseteq d(L)$, for some $n>1$, then $L$ is solvable.

2010 Mathematics subject classification: primary 17B40.

Keywords and phrases: Lie algebras, derivations, solvable Lie algebras, compact Lie groups.
\end{abstract}

In [3], Ladisch proved that a finite group $G$, admitting an element $a$ with the property $G^{\prime}=[a, G]$, is solvable. Here, $[a, G]$ is the set of all commutators $[a, x]$, with $x \in G$. Using this result, one can prove that a finite group is solvable if it has a fixed point free automorphism. In this note, we prove a similar result for Lie algebras in a more general framework; we show that a finite-dimensional Lie algebra $L$ of characteristic zero is solvable if it has an abelian subalgebra $A$ with the property $L^{n} \subseteq[A, L]$, for some $n>1$, where $[A, L]$ denotes the linear subspace generated by all commutators of the form $[a, x]$, with $a \in A$ and $x \in L$. Next, we use this result to prove that a finitedimensional Lie algebra $L$ over a field of characteristic zero, admitting an abelian algebra of derivations $D \leq \operatorname{Der}(L)$ with the property

$$
L^{n} \subseteq \sum_{d \in D} d(L)
$$

for some $n>1$, is necessarily solvable. As a special case, we conclude that if the Lie algebra $L$ admits a derivation $d: L \rightarrow L$, such that $L^{n} \subseteq d(L)$, for some $n>1$, then $L$ is solvable. Note that a similar result was obtained by Jacobson in [2]: a finite-dimensional Lie algebra over an algebraically closed field of characteristic zero, admitting an invertible derivation, is nilpotent.

Our main result (Corollary 2 below) is also true for connected compact Lie groups, and so it may be also true for finite groups. Therefore, we ask the following question.

(C) 2011 Australian Mathematical Publishing Association Inc. 0004-9727/2011 \$16.00 
Let $G$ be a finite group admitting an abelian subgroup $A$ with the property $\gamma_{n}(G) \subseteq$ $\{[a, x]: a \in A, x \in G\}$, for some $n>1$. Is it true that $G$ is solvable?

In this note, $L$ is a finite-dimensional Lie algebra over a field $K$ of characteristic zero. We denote by $L^{n}$ and $L^{(n)}$ the $n$th terms of the lower central series and derived series of $L$, respectively. Also we denote by $\operatorname{Der}(L)$ the algebra of derivations of $L$.

THeOREM 1. Let L be a finite-dimensional Lie algebra over $K$ and suppose that $U$ is an ideal. Suppose that there is an abelian subalgebra A such that $U^{n} \subseteq[A, U]$, for some $n>1$. Then $U$ is solvable.

Proof. First, we assume that $K$ is algebraically closed, so we can apply the solvability criterion of Cartan. Let $S=U^{n}$ and define a symmetric bilinear form on $L$ by

$$
\kappa_{S}(x, y)=\operatorname{Tr}\left(a d_{S} x \circ a d_{S} y\right),
$$

where $a d_{S}$ denotes the restriction of $a d$ on $S$. Note that, since $S$ is an ideal of $L, \kappa_{S}$ is associative, that is,

$$
\kappa_{S}([x, y], z)=\kappa_{S}(x,[y, z]) .
$$

We show that $\kappa_{S}\left(S, S^{\prime}\right)=0$. However, since $S^{\prime} \subseteq U^{n} \subseteq[A, U]$, we prove that $\kappa_{S}(S,[A, U])=0$. By the associativity of $\kappa_{S}$, this is equivalent to $\kappa_{S}([S, U], A)=0$. But we have $[S, U] \subseteq U^{n} \subseteq[A, U]$, therefore it is enough to prove that $\kappa_{S}([A, U], A)=0$. By the associativity again, this is just $\kappa_{S}([A, A], U)=0$, which is true, because $A$ is abelian. Hence $S$ is solvable by Cartan's criterion and, since $U^{(n-2)} \subseteq U^{n-1}=S, U$ is solvable.

We now suppose that $K$ is not necessarily algebraically closed. Let $\bar{K}$ be its algebraic closure and $\bar{L}=\bar{K} \otimes_{K} L$. Now $\bar{L}$ is a finite-dimensional Lie algebra over $\bar{K}$ in which $\bar{K} \otimes_{K} U$ is an ideal and $\bar{K} \otimes_{K} A$ is an abelian subalgebra. Further, we have

$$
\left(\bar{K} \otimes_{K} U\right)^{n}=\bar{K} \otimes_{K} U^{n} \subseteq\left[\bar{K} \otimes_{K} A, \bar{K} \otimes_{K} U\right] .
$$

So $\bar{K} \otimes_{K} U$ is solvable, that is, there is a number $m$ such that $\left(\bar{K} \otimes_{K} U\right)^{(m)}=0$. On the other hand,

$$
\left(\bar{K} \otimes_{K} U\right)^{(m)}=\bar{K} \otimes_{K} U^{(m)},
$$

therefore $U^{(m)}=0$.

As a result, if we assume that $U=L$, we obtain the following corollary.

Corollary 2. Suppose that there exist an abelian subalgebra $A \leq L$ and an integer $n>1$, such that $L^{n} \subseteq[A, L]$. Then L is solvable.

As another result, we have the following corollary.

Corollary 3. Suppose that $L$ is semisimple and $A$ is an abelian subalgebra. Then $[A, L] \varsubsetneqq L$.

Using the Lie functor, we can restate Corollary 2 for connected compact Lie groups.

Corollary 4. Suppose that a connected compact Lie group $G$ has an abelian Lie subgroup $A$, such that $\gamma_{n}(G) \subseteq\{[a, x]: a \in A, x \in G\}$, for some $n>1$. Then $G$ is solvable. 
Finally, we can apply Theorem 1 to derivations of Lie algebras to obtain a sufficient condition for solvability.

Corollary 5. Suppose that there exist an abelian subalgebra $D \leq \operatorname{Der}(L)$ and an integer $n>1$ such that

$$
L^{n} \subseteq \sum_{d \in D} d(L)
$$

Then L is solvable.

Proof. Let $\hat{L}=D \ltimes L$, the natural semidirect product. Then $L$ is an ideal and $D$ is an abelian subalgebra in $\hat{L}$. Note that in the semidirect product,

$$
[D, L]=\sum_{d \in D} d(L),
$$

hence the assumption is just $L^{n} \subseteq[D, L]$. So, $L$ is solvable by Theorem 1 .

As a special case, if the Lie algebra $L$ admits a derivation $d: L \rightarrow L$ such that $L^{n} \subseteq d(L)$, for some $n>1$, then $L$ is solvable. Note that our results are not true for Lie algebras of positive characteristics, since there are simple Lie algebras over fields of nonzero characteristics, that admit invertible derivations; see, for example, [1].

\section{Acknowledgements}

The author would like to thank P. Shumyatski and K. Ersoy for their comments. He would also like to thank the referee for his/her invaluable suggestions.

\section{References}

[1] G. Benkart, A. I. Kostrikin and M. I. Kuznestov, 'Finite dimensional Lie algebras with a nonsingular derivation', J. Algebra 171 (1995), 894-916.

[2] N. Jacobson, 'A note on automorphisms and derivations of Lie algebras', Proc. Amer. Math. Soc. 6 (1955), 281-283.

[3] F. Ladisch, 'Groups with anti-central elements', Comm. Algebra 36 (2008), 2883-2894.

\section{SHAHRYARI, Department of Pure Mathematics,}

Faculty of Mathematical Sciences, University of Tabriz, Tabriz, Iran

e-mail: mshahryari@tabrizu.ac.ir 